Research Note

\title{
Molecular Orbital Calculations for Dioxetane as a Part of the Intermediate of Firefly Luciferin
}

\author{
Shinichi ITOH and Naoyosi NAMEDA
}

Department of Information and Computer Science, University of Kagoshima

1-21-40 Korimoto, Kagoshima-shi, 890 Kagoshima, Japan

\begin{abstract}
Firefly luciferin $(\mathrm{Ln})$ is an absorbing substance which showing a high quantum efficiency of the bioluminescence. Several proposals about the mechanism of the luminescence in this system have been explained by investigators. A dioxetane structure exists within the intermediate of $L n$ and plays an important role to occure the emission. In this report, we attempt to calculate the electronic structures of the dioxetane and to correlate the calculated results with the experimental observations as a first step to recognize the mechanism of the bioluminescence of $\mathrm{Ln}$. Obtained results show that the $2 p$ electrons of $\mathrm{O}$ and $\mathrm{C}$ are important as to the electronic excitations of the luminescence. The calculated excitation energy agrees with the observed value in spite of a simplified system.
\end{abstract}

\section{Introduction}

Firefly luciferin ( $\mathrm{Ln})$ is an attractive chemical material since $\mathrm{Ln}$ emits strong visible ray owing to the much high quantum efficiency of the bioluminescence[1]. The strong bioluminescence occures by the chemical reaction of $\mathrm{Ln}$ and the enzyme luciferase with ATP, $\mathrm{O}_{2}$ and $\mathrm{Mg}^{2+}$ (Fig. (1)). As the results, the tetragonal ring peroxide (dioxetane structure, the first arrow of Fig. (1)) is generated and succesively changes to oxyluciferin with the strong emission [2]. The dioxetane structure is the intermediate in the process of yielding the excited oxyluciferin. Thus, considering the importance of dioxetane structure to the bioluminescence we have tried to perform the semiempirical molecular orbital (MO) calculation to the tetragonal ring of dioxetane structure Fig. (2). The purpose of this resport is to convince the performance of the present MO calculation for the sake of study of the machanism of the bioluminescence of $\mathrm{Ln}$ in the further step.
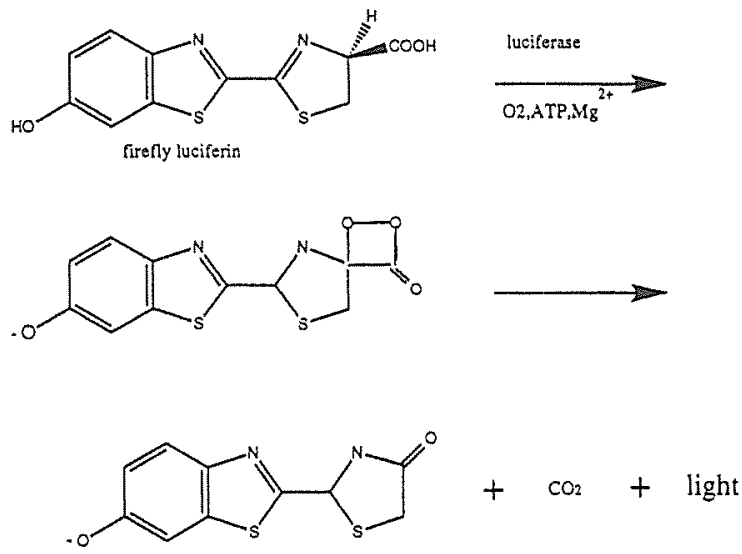

Fig. 1 Firefly luciferin, intermediates and chemiluminescent material. The intermediate includes the dioxyetane.

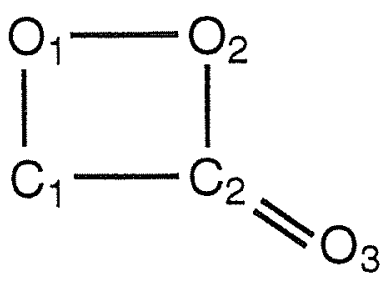

Fig. 2 The schematic molecular structure of the tetragonal ring peroxide (dioxetane structure) with the atomic labels.

\section{Method of Calculation}

\section{A. Calculation of the Energy of Molecular Orbitals}

We used the program package MOPAC93 for the MO calculations. By solving the simultaneous equation Eq.(1), the MOs and their energies were obtained.

$$
\begin{aligned}
& \mathrm{FC}=\mathrm{C} \varepsilon_{\mathrm{D}}, \\
& \mathrm{C}^{\mathrm{T}} \mathrm{C}=\mathrm{I} .
\end{aligned}
$$

Here, $\mathbf{F}$ is Fock-matrix, $\mathbf{C}$ and $\varepsilon_{\mathrm{D}}$ are coefficients of MOs and their energies, respectively. Next Eq.(2) is the additional condition as to the normalization of the MOs. In the present calculations, the Fock matrix called MNDO PM3 is adopted, in which 18 kinds of parameters (Coulomb repulsion, size of atomic orbitals, nuclear repulsion and so on) exist for an atom, respectively. The above secular equations are solved by the self-consistent-field (SCF) approach. 


\section{B. Estimation of the Excitation Energy}

By using the obtained one-electron energy levels and total energies, we estimate the excitation energy of dioxetane structure. Since oxyluciferin are yielded following to the excitation of dioxetane, the excitation energy of dioxetane structure is meaningful to compared with the energy of the luminescence. We adopted the two formalisms to calculate the excitation energy of the system. One is to calculate the difference of the one-electron MO energies between the lowest unoccupied molecular orbital (LUMO) and the highest occupied molecular orbital (HOMO). In the ground state of dioxetane structure, each of MOs from in the bottom level up to in the $i$-th level (HOMO) are occupied by the two electrons. When one of the electrons in the $i$-th level moves to the upper $i+1$-th level (LUMO), the excitation of the system occures. Then we calculate,

$$
\Delta \varepsilon=\varepsilon_{i+1 . \mathrm{gr}}-\varepsilon_{i, \mathrm{gr}},
$$

where $\varepsilon_{i, g r}$ is the energy of the $i$-MO in the ground state. The other procedure is to compare the total energies both in the ground state and in the excited state.

$$
\Delta E_{\mathrm{tot}}=E_{\mathrm{tot}, \mathrm{exc}}-E_{\mathrm{tot}, \mathrm{gr}}
$$

which is called $\triangle S C F$ method. We estimated the excitation energies by these procedures.

\section{Results}

The bondlengths and the bond angles of the dioxetane structure are listed in Table. I. With the use of this structure, the one-electron MO energies were calculated and specified in Fig. 3 nearby HOMO and LUMO. In the semiempirical Hartree-Fock calculation, only the valence orbitals of atoms are explicitely solved. As is mentioned above, since the emission is caused by the electronic excitations in the HOMO and LUMO, the energy levels of the two MOs should be considered to be important about the emission. In the ground state, each of the MOs below the HOMO are doubly occupied and the LUMO is empty. The excited state was calculated by the configuration of singly occupied HOMO and LUMO with the spin singlet state. The obtained one-electron energies are $1.235(\mathrm{eV})$ for HOMO and $2.852(\mathrm{eV})$ for LUMO in the ground state. Then, we obtained the excitation energy by Eq. (3) as 1.617 (eV).
The total energies are $-1111.04029(\mathrm{eV})$ for the ground state and $-1108.90906(\mathrm{eV})$ for the singlet excited state, then, we obtained the excitation energy by Eq. (4) as $2.13123(\mathrm{eV})$. In Table II, the calculated energies and the experimental observation are listed. It seems that our results gave close energies to the experiment. The calculated energies are in the region of the visible ray. This agrees with the bioluminescence of firefly. Furthermore, in order to investigate the relationship between the electronic structure and the bioluminescence, the atomic character of MOs is shown in Table III. The dominant atomic characters are C$2 p$ and $\mathrm{O}-2 p \sigma$-orbitals. It is noted that the HOMO and LUMO are antibonding orbitals. This suggests that the HOMO of dioxetane is unstable and the possibility of the decomposition of this dioxetane structure into several materials, which agrees with the case of the chemical reaction $\mathrm{Ln}$ to oxy-Ln.

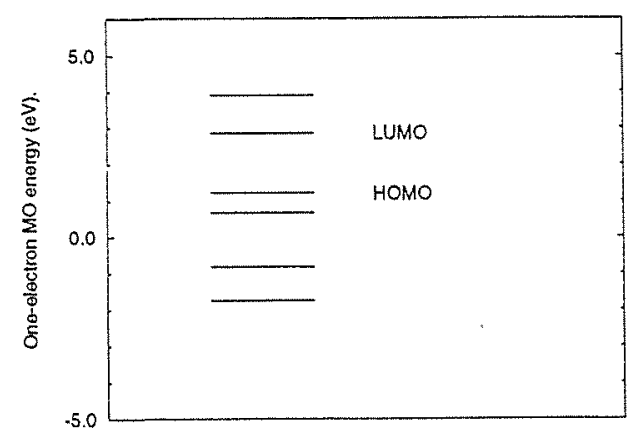

Fig. 3 One-electron energy of the dioxyetane nearby HOMO and LUMO in the ground state. The total energy is $-1111.04029(\mathrm{eV})$.

Table I Atomic structure of dioxetane structure (in $\AA$ for bond lengths and degrees for bond angles.)

\begin{tabular}{ll|ll}
\hline \hline \multicolumn{2}{c|}{ Bond lengths } & \multicolumn{2}{c}{ Bond angles } \\
\hline $\mathrm{C}_{1}-\mathrm{C}_{2}$ & 1.52 & $\mathrm{C}_{1}-\mathrm{C}_{2}-\mathrm{O}_{2}$ & 90.0 \\
$\mathrm{C}_{2}-\mathrm{C}_{3}$ & 1.16 & $\mathrm{C}_{1}-\mathrm{C}_{2}-\mathrm{C}_{3}$ & 135.0 \\
$\mathrm{C}_{2}-\mathrm{O}_{2}$ & 1.36 & $\mathrm{O}_{1}-\mathrm{O}_{2}-\mathrm{C}_{2}$ & 87.26 \\
$\mathrm{O}_{1}-\mathrm{O}_{2}$ & 1.48 & & \\
\hline \hline
\end{tabular}

Table II Excitation energies of the dioxetane structure.

\begin{tabular}{rll}
\hline \hline & $(\mathrm{eV})$ & $\left(10^{3} \mathrm{~cm}^{-1}\right)$ \\
\hline By Eq.(3) & 1.62 & 13.05 \\
By Eq.(4) & 2.13 & 17.18 \\
Exp.[1] & 2.73 & 22.01 \\
\hline \hline
\end{tabular}

Tabl III Atomic orbitals in HOMO and LUMO in ground state.

\begin{tabular}{rrrrrrrrrrr}
\hline \hline Atom & \multicolumn{2}{c}{$\mathrm{C}_{1}$} & \multicolumn{2}{c}{$\mathrm{C}_{2}$} & \multicolumn{2}{c}{$\mathrm{O}_{1}$} & \multicolumn{2}{c}{$\mathrm{O}_{2}$} & \multicolumn{2}{c}{$\mathrm{O}_{3}$} \\
$\mathrm{AO}$ & $2 s$ & $2 p$ & $2 s$ & $2 p$ & $2 s$ & $2 p$ & $2 s$ & $2 p$ & $2 s$ & $2 p$ \\
\hline LUMO & 0.069 & 0.302 & 0.001 & 0.297 & 0.012 & 0.094 & 0.000 & 0.064 & 0.032 & 0.149 \\
HOMO & 0.023 & 0.330 & 0.320 & 0.033 & 0.020 & 0.164 & 0.008 & 0.074 & 0.000 & 0.029 \\
\hline \hline
\end{tabular}




\section{Conclusion}

We performed semi-emperical Hartree-Fock calculations for the dioxetne which is a part of firefly luciferin. HOMOLUMO gap and $\triangle \mathrm{SCF}$ estimations showed the close value to the bioluminescence in the visible region.

\section{Acknowledgements}

We thank the Computer Center, Institute of Molecular Science, Okazaki National Research Institutes for the use of HSP computer.

[1] T. Hirano and M. Ohashi,: "Kagaku to seibutu 31", 195 (1993)

[2] N. Wada, M. Honda and H. Suzuki,: "J. Phys. Soc. Jpn 54" 4851 (1985). 